\title{
EL ADJETIVO CALIFICATIVO ESPAÑOL Y SU POSICIÓN DENTRO DEL SINTAGMA NOMINAL
}

\begin{abstract}
Pawlik Janusz, El adjetivo calificativo Español y su posición dentro del sintagma nominal [The Spanish qualifying adjective and its position in a noun phrase], Studia Romanica Posnaniensia, Adam Mickiewicz University Press, Poznan, vol. XXVII: 2001, pp. 129-141. ISBN 83-232-1039-X, ISSN 0137-2475.

With this little paper we intend to draw the attention of Polish speakers of Spanish to the problem of word order of qualifying adjectives in a noun phrase. In our opinion, this grammatical issue should be tackled successfully only by carrying out an in-depth semantic study of the contents of these noun determiners. For this purpose we are putting forward a general semantic classification of this category of words demonstrating that the meaning of a given item is closely connected with its syntactic position.
\end{abstract}

Los adjetivos de esta clase describen al sustantivo informándonos de alguna propiedad interna o externa del referente. Los calificativos forman una clase muy numerosa de palabras léxicas. En tal sentido se oponen a los adjetivos determinativos, que forman una clase cerrada de palabras gramaticales. Desde el punto de vista de su comportamiento sintáctico, distinguimos en los calificativos dos empleos distintos. Llamamos adjetivo atributivo al que modifica al sustantivo directamente, como: el libro blanco, y adjetivo predicativo, al que lo hace por medio de un índice verbal, es decir, indirectamente: el libro es blanco.

En español, el adjetivo atributivo tiene libertad de colocación en la frase y, por eso, vamos a prestarle particular atención. Al abordar este difícil problema hay que constatar que comparado con el francés es un tema menos estudiado y, tal vez, menos comprendido por los hablantes extranjeros, en especial, procedentes de fuera de la zona románica. Bien es cierto que el orden de colocación de elementos modificadores en un SN se atiene a algunos preceptos universales y no se restringe tan solo al ámbito hispánico. Por lo tanto, la buena comprensión del problema resultaría doblemente útil tanto más cuanto que la sintaxis del adjetivo en un SN obedece, en líneas generales, al mismo principio en otras lenguas romances.

Huelga decir que en estos idiomas, la determinada posición de un adjetivo calificativo va asociada al diferente matiz estilístico que se le imprime. Más aun, no 
es raro que, algunas veces, llegue a alterar el contenido semántico del propio adjetivo. En vista de ello, el punto de arranque y la base continua de referencia en nuestra investigación, por somera que sea, debe ser el contenido semántico de la frase nominal. Como es sabido, la ordenación de adjetivos polacos dentro del SN es bastante fija o, al menos, mucho más rigurosa que en castellano. El fin primordial será, entonces, tratar de impedir la interferencia de nuestra lengua nativa, y rastrear unos principios que estén al origen de la configuración tan caprichosa de los adjetivos en la lengua española.

Cuanto llevamos dicho hasta ahora es válido sólo en parte, ya que ni el polaco ostenta el orden de complementos referidos al nombre absolutamente fijo, ni en español nos encontramos con la sucesión de dichos elementos totalmente suelta. Para empezar, es preciso partir de la consideración detenida de la enorme clase de adjetivos calificativos. Aplicando un enfoque semántico, se constata que tanto en español como en otras lenguas indoeuropeas, las unidades gramaticales en cuestión se pueden repartir en dos tipos fundamentales y bien distintos. Los primeros son los adjetivos de origen (cuantitativos), esto es, aquellos que designan cualidades del nombre y no se derivan de otras partes del discurso. Ordinariamente dan respuesta a la pregunta ¿cómo es?, y como cualquier adjetivo por antonomasia admite la variación del grado, como en alto / más alto / el más alto; wysoki / wyższy / najwyższy (Buttler, Kurkowska, Satkiewicz 1986: 392-393). Para ilustrarlo veamos algunos ejemplos:

bonita mujer - ladna kobieta,

hombre delgado - szczupty meżczyzna,

importante asunto - waina sprawa,

sentido profundo - głęboki sens.

En polaco, los adjetivos de origen van normalmente antepuestos al nombre y son rarísimos los casos de infracción a esta regla. El adjetivo lleva de ordinario su significado literal y su colocación estable no está vinculada a factores semánticos algunos. En el idioma español, por el contrario, nos encontramos aproximadamente con el mismo número de unidades pospuestas como antepuestas en una frase nominal. Su posición es generalmente significativa, o sea, está dotada de matices estilísticos o semánticos adicionales. Al contrario de la opinión corriente, el polaco asimismo es capaz de expresar, en ocasiones, contenidos distintos mediante dicho recurso. Comparemos:

biedny człowiek - pobre hombre, człowiek biedny - hombre pobre,

pewna sprawa - cierto asunto,

sprawa pewna - asunto cierto.

La segunda gran clase de unidades que conviene oponer a la primera, se halla constituída por los adjetivos derivados de otro origen. Se trata, en primer lugar, de 
sustantivos, raras veces verbos, y los adjetivos formados a partir de esta fuente pueden parafrasearse mediante un sintagma preposicional con la base nominal del mismo adjetivo. Normalmente, contestan a la pregunta ¿qué tipo? y, en efecto, guardan con el nombre la relación del género a la especie. De ahí que se han venido en llamar adjetivos relacionales (no cuantitativos). Así, por ejemplo (Moreno 1993: 251):

viaje espacial $=$ viaje al espacio.

asunto nacional = asunto de la nación.

problema económico $=$ problema de la economía .

ataque cardíaco = ataque al corazón.

\section{LOS ADJETIVOS RELACIONALES (NO CUANTITATIVOS)}

Además de su carácter nominal y genérico, su rasgo particular es la incapacidad de expresar el grado, o sea, ni en polaco ni en español estos adjetivos modifican al sustantivo con una intensidad medible de la propiedad denotada. Al contrario, al igual que el nombre del que procede no se someten a la variación de este accidente gramatical. Véase la agramaticalidad de las secuencias:

*campeonato más mundial - *mistrzostwa bardziej światowe,

*industria menos maderera - *przemyst mniej drzewny.

Como sabemos, el campeonato puede ser mundial o no, igual que la industria puede ser maderera o no, y no hay soluciones intermedias. Semánticamente, dan a entender que existe una relación entre dos sustantivos y no matizan su extensión. Por igual razón, el adjetivo relacional no admite adverbios enfáticos que acentúen la propiedad que significa (Zierer 1974: 57). De ahí que sean censurables en lenguaje corriente frases como:

*la historia muy universal,

* un ataque tan cardíaco,

*el comité extremamente internacional.

Este tipo de adjetivo aparece con bastante regularidad en posición postnominal en polaco, y en español éste es su lugar obligatorio. Por lo tanto, en cuanto a los adjetivos mencionados no se puede hablar de su libertad de orden, y mucho menos en castellano. Consideremos otros ejemplos:

máquina calculadora - *calculadora máquina,

crisis económica - *económica crisis,

industria textil - *textil industria,

país democrático - *democrático país.

En tales ejemplos, que sería fácil multiplicar, la anteposición del adjetivo supondría una figura estilística extremamente violenta, solo admisible en lenguaje poético. 
Lo mismo ocurre con los adjetivos verbales, es decir, participios pasivos y algunos gerundios que funcionan como verdaderos adjetivos. Aquí, el adjetivo polaco precede y el español sigue normalmente al núcleo del sintagma en lenguaje cotidiano. Comparemos:

el trabajo terminado - skończona praca,

el libro comprado - kupiona ksiażka!,

agua hirviendo - wrzqca woda,

los brazos colgando-zwisajace ramiona.

Es de añadir que en los adjetivos verbales, así como en los nominales, no existe graduación de la intensidad ni de la cantidad de la propiedad designada. Por eso, serían muy insólitas frases españolas ${ }^{2}$, como:

*el libro más comprado,

*el trabajo menos terminado,

*el agua muy hirviendo.

Así, de todo lo anterior se concluye que, en español, la regularidad de posponer este tipo de adjetivos al nombre es absoluta y afecta a todos los registros del lenguaje. En la lengua polaca, en cambio, el empleo de los adjetivos relacionales pospuestos al sustantivo es propio más bien del lenguaje escrito, periodístico o científico. Sin embargo, como ya hemos comprobado, algunos de ellos van antes del nombre y son característicos también para el habla más coloquial, p.ej.: żelazny krzyż; drewniany stót; zepsuty telewizor.

La cosa se va complicando cuando un mismo sustantivo aparece acompañado de varios modificadores de la clase examinada. Aquí se plantea el problema de la ordenación de adjetivos entre sí, porque si todos deben seguir al nombre, habrá, tal vez, algunos principios que regulen su correlación mutua dentro del SN. Hay algunas predisposiciones intrínsecas según que un adjetivo aparezca en un SN más cerca o más lejos del nombre. De entre muchos conceptos a los que pueden aludir los adjetivos relacionales destacan nacionalidad, situación geográfica o locativa. No sería arriesgado constatar que las unidades que lo significan suelen ocupar el último lugar en la serie. Así, p.ej.:

Comunidad Económica Europea,

Partido Socialista Obrero Español,

Partido Obrero Unificado Polaco,

gobierno demócrata norteamericano,

Comité Olímpico Internacional.

${ }^{3}$ El participio se desplaza detrás del nombre si va acompañado de complementos, p.ej.: ksiqżka kupiona w naszym sklepie.

${ }^{2}$ Raras veces, el participio pasivo pierde su carácter verbal, convirtiéndose en adjetivo cuantitativo, p. ej. técnica muy avanzada; su avanzada tecnología. 
Igual que en la sintaxis oracional, el complemento circunstancial de lugar viene a colocarse ordinariamente al final del enunciado, también en las frases citadas, la información referente a la procedencia o extensión espacial del núcleo va después de todos los modificadores genéricos. El orden de colocación tampoco se ve perturbado cuando en lugar de otro adjetivo aparece un complemento preposicional de+nombre. Así: secretario de estado norteamericano; hablante de español polaco. Con las estructuras anteriores damos a entender que hay otros secretarios de estado que no son norteamericanos, y que existen otros hablantes de español además de los polacos. Desde el punto de vista lógico cualquier adjetivo pospuesto delimita o restringe la extensión del sustantivo; si decimos Comité Olímpico Internacional, excluimos de la imagen general de comité a todos aquellos que no sean internacionales, y efectivamente existen comités olímpicos nacionales. Por otra parte, consideremos el nombre del Comité Internacional de Auschwitz, donde el adjetivo internacional que designa extensión geográfica o nivel de actividad viene antes del complemento preposicional de Auschwitz. De lo anterior se puede suponer, aunque sin absoluta seguridad, que este organismo no tiene otros parecidos a nivel inferior, esto es, regional o nacional, y no forma una red de instituciones que se puedan oponer unas a otras.

La correlación locativa de adjetivos relacionales puede obedecer también a razones de carácter rítmico y lo normal es que un elemento corto preceda a uno más largo en una serie, como en:

\section{soldado negro norteamericano.}

Adviértase que aquí negro se refiere a la raza y se sitúa en el mismo nivel categorial semántico que norteamericano. En este caso, el orden natural es que los adjetivos de raza, como concepto más amplio, precedan a los de nación. Sin embargo, nada impide que sea gramatical también la frase con el orden de los calificativos inverso soldado norteamericano negro.

En cuanto a otros tipos de adjetivos, debemos seguir el principio de que el nombre se une en primer lugar con unidades que le adjudican una propiedad que le es más natural. Se trata de adjetivos que expresan con el sustantivo una noción entera e indivisible, como si se tratase de una frase hecha bien arraigada en la lengua. Pongamos un ejemplo:

seguro social agrícola $*$ seguro agrícola social.

La frase seguro social funciona en la lengua y el adjetivo agrícola sólo modifica este conjunto, mientras que el grupo seguro agrícola puede difícilmente ser modificado por social. Ahora examinemos dos sintagmas donde un mismo adjetivo se coloca distintamente según el sustantivo a que se refiere:

Juventud Obrera Socialista,

Partido Socialista Obrero. 
Por ciertas razones, el sustantivo partido se aproxima más al término socialista que el sustantivo juventud. En otras palabras, la frase juventud obrera tiene prioridad ante juventud socialista, es decir, la juventud puede ser, en primer lugar, obrera y así se caracteriza mejor, y sólo después socialista. Por el contrario, el partido, en cuanto organización política, es por su definición socialista o no, de ahí que ambos términos sean más afines. Sólo después, los conceptos de partido socialista y juventud obrera pueden delimitarse por otros atributivos, es decir, respectivamente obrero y socialista. De forma análoga, podemos interpretar la posición inestable del adjetivo obrero. Veamos cómo lo que queda dicho hasta el momento se puede apreciar en otros ejemplos:

\section{Asistencia Recíproca Petrolera Estatal Latinoamericana, Juventud Obrera Cristiana Internacional, Líneas Aéreas Europeas Británicas, Asociación Estudiantil Democrática.}

\section{LOS ADJETIVOS CUANTITATIVOS (DE ORIGEN)}

Volvamos ahora a la gran clase de adjetivos cuantitativos, estudiando detenidamente su carácter, comportamiento y ordenación dentro del SN. A diferencia de los relacionales, son unos adjetivos originales y susceptibles de experimentar la gradación. Los encontramos predominantemente en la lengua corriente y literaria, y su libertad de posición frente al nombre deja perplejos a muchos hablantes polacos.

De un modo general, el adjetivo de este tipo puede seguir o proceder al sustantivo a que se refiere. Desde el punto de vista de la corrección gramatical nada se opone a que digamos nubes blancas o blancas nubes, saludo afectuoso o afectuoso saludo. Pero la forma interior del lenguaje que nos hace preferir una u otra colocación en cada caso concreto está regulada por factores lógicos, estilísticos y rítmicos, que actúan conjuntamente a manera de tendencias, y motivan que no sea siempre indiferente el lugar que ocupa este calificativo. Según ya se apuntaba, el adjetivo pospuesto señala una especificación que restringe la referencia propia del sustantivo. Al decir, por ejemplo, pared blanca denotamos una realidad particular opuesta a otras posibles (pared azul, pared verde, etc.) especificando así la realidad concreta de la clase de objetos pared a que se alude. Sin embargo, el valor de la anteposición y la posposición del adjetivo depende muchas veces de la intención del hablante más que de criterios objetivos. Así, el adjetivo prenominal revela una intención explicativa y descriptiva de la realidad sugerida por el sustantivo, y por ello se ha dado en llamar adjetivo explicativo o epíteto. En cambio, el adjetivo postnominal realiza la función especificativa y restrictiva de la significación del núcleo, y aquí reside su carácter de adjetivo especificativo. 
Por esto, resultaría chocante la posposición de un adjetivo que signifique cualidades inseparablemente asociadas a la imagen del sustantivo, como las ovejas mansas o miel dulce, ya que no podemos imaginar ovejas que no sean mansas o miel que no sea dulce. En la blanca nieve, p. ej., no se designa a ningún tipo de nieve que se oponga a otro que no sea blanco, sino que simplemente se describe cómo es la nieve.

\subsection{LOS SN CON UN SOLO ADJETIVO}

Bien que son muchos los casos en que un núcleo nominal cuenta con más cuantitativos, sería aconsejable empezar el análisis por el grupo más sencillo. Veamos, al principio, grupos nominales donde, además de un adjetivo, aparece un posesivo:

\section{tu bonita mujer,}

su acusado sentido del deber,

mi admirable marido,

el libro y su elevado precio,

el mendigo y sus flacas manos.

Obviamente, en el primer ejemplo, el poseedor no puede tener más que una mujer. Teniendo una bonita, lógicamente, no se puede oponerla a otras que no sean bonitas. Por eso, la expresión *tu mujer bonita resultaría insólita. Ocurre lo mismo en los ejemplos restantes donde el orden no marcado que se impone es posesivo + adjetivo + nombre. Las pocas infracciones a este principio se explicarán, entre otros, por motivos rítmicos; el adjetivo con más sílabas que el nombre puede seguirle usualmente, p.ej.: su carácter excepcional. En la misma construcción, se admite la posposición del adjetivo cuando podemos imaginar que el concepto designado puede tener varias realizaciones. Lo apreciamos, por ejemplo, en:

\section{el idioma y su correcta utilización / el idioma y su utilización correcta.}

Es evidente que la utilización del idioma en cada hablante puede ser correcta o no; así resaltamos y hacemos valorar esta propiedad frente a otras. El dejar el adjetivo antepuesto le imprime al conjunto un carácter más afectivo, pero podemos considerar como sinónimas las dos expresiones. Notemos que la estructura:

el idioma y su correcta utilización

se puede transformar en otra equivalente:

la correcta utilización del idioma / la utilización correcta del idioma

que lleva idéntica información. Aunque la permutación del adjetivo de estas secuencias produzca ligeros cambios de matiz estilístico (no lógico) en su sentido global, según se indicó al tratar de la posposición y la anteposición, el resultado no 
perturba la estructura del grupo ni la relación entre sus componentes. Entonces, sería lo mismo decir también:

la desastrosa historia de Cayo Graco,

la grosera observación del maestro

que

la historia desastrosa de Cayo Graco,

la observación grosera del maestro.

Por otra parte, sería censurable la frase *la muerte trágica de José Soto en vez de la trágica muerte de José Soto, dado que cualquier muerte es trágica y es redundante su especificación. Tampoco estaría bien la frase *el común sentido de Carmen por la simple razón de que el uso ha fijado la combinación sentido común como si fuese un sustantivo compuesto.

Es digno de reflexión el caso de grupos parecidos con un nombre en plural, como:

las hojas secas del parque se cayeron / las secas hojas del parque se cayeron.

Aquí, la variación de posición experimentada por el adjetivo arrastra una modificación semántica en el enunciado. La posposición supone que sólo las hojas secas se cayeron, y otras no. La anteposición, por el contrario, significa que todas las hojas se cayeron porque todas eran secas. Por esta razón, resulta disparatada la secuencia siguiente: *las arrugas venerables de su frente, por ser contraria a la realidad. Es inimaginable que una misma frente tenga unas arrugas venerables y otras no. Su oposición es, entonces, incompatible con la lógica. Estas lecturas distintas hacen que por la misma razón se pueda aceptar la frase:

todos los puntos oscuros de este texto

mientras que puede resultar extraña otra:

todos los oscuros puntos de este texto

que sugiere que en el texto no hay puntos claros, lo que raramente coincide con la realidad. Más aun, la última frase parece ser propia del lenguaje poético, donde el adjetivo oscuro viene a adquirir un sentido figurado de misterioso, siniestro, demoniaco. Esto supuesto, hay que tener siempre cuidado al ajustar el lugar del modificador a nuestra intención comunicativa.

La distinción entre epítetos y adjetivos especificativos se concreta a veces en la diversidad de significación. Aquí asimismo, el sentido recto se conserva en el elemento postnominal, en tanto que en el prenominal se halla más o menos deformado. Cotéjense los sentidos del adjetivo en estos casos opuestos:

triste funcionario (=humilde),

funcionario triste (=apenado), 
gran cuadro (=excelente),

cuadro grande (=enorme),

simple soldado (=sencillo, sin graduación),

soldado simple (=tonto),

rara noticia (=poco frecuente),

noticia rara (=extraña).

Conviene señalar que los adverbios antepuestos a un adjetivo completan la significación de éste. En esta función complementaria se usan principalmente los adverbios de cantidad y de modo: muy, más, tan, extremamente, bastante, etc. En español, contrariamente al polaco, el conjunto sintagmático adverbio + adjetivo va siempre detrás del nombre, cualquiera que sea el tipo semántico del adjetivo calificativo. Comparemos:

paseo muy largo / bardzo dhugi spacer,

problema extremamente dificil / niezwykle trudny problem, cuestión tan amplia / tak(a) szeroka kwestia,

edificio más alto / wyższy budynek.

Salvadas estas restricciones, generalmente el adjetivo cuantitativo puede variar libremente de posición en la mayoría de los casos. Pongamos algunos ejemplos de esta permutación en sintagmas de un solo adjetivo ${ }^{3}$ :

una clara alusión al nazismo / una alusión clara al nazismo,

estas fatídicas palabras / estas palabras fatídicas,

un amplio entendimiento entre médicos y pacientes / un entendimiento amplio, entre médicos y pacientes,

con enormes dificultades / con dificultades enormes,

un maravilloso crucero / un crucero maravilloso,

el rojo sombrero de Juan / el sombrero rojo de Juan.

Téngase presente que en las secuencias de la izquierda, el adjetivo presenta la cualidad como algo inherente a la persona o cosa en cuestión, por lo que ofrece tanto interés en el estilo literario. En la siguiente nota publicitaria, se puede apreciar cómo el término que se anticipa denota actitud valorativa y afectiva de su autor ${ }^{4}$ :

${ }^{3}$ Algunos adjetivos aparecen más frecuentemente en una posición que en otra. Tal es el caso de ligero. Así, en sentido figurado se suele anteponer, como en: con ligera expresión de fastidio, un ligero descenso en las bodas celebradas, y en su sentido literal suele seguir al nombre, p.ej.: el corredor y su bicicleta ligera.

${ }^{4}$ Este pequeño texto se podría redactar, por supuesto, de forma más moderada y menos afectada, es decir: El buque ofrece un servicio esmerado del que podemos disfrutar a lo largo de un crucero maravilloso de ocho dias inolvidables. 
El buque ofrece un esmerado servicio del que podemos disfrutar a lo largo de un maravilloso crucero de ocho inolvidables dias.

Para terminar, conviene distinguir una subclase semántica de adjetivos que agrupa a los llamados modales (posible, necesario, probable, presunto, supuesto, presumible, dudoso, indudable, etc.) que tienden a fijarse en un orden determinado, precediendo obligatoriamente al núcleo (Demonte 1998). Así: el presunto asesino, la supuesta declaracion, un posible fracaso, etc. También el adjetivo mero toma regularmente la posición prenominal: la mera opinión, la mera semejanza.

\subsection{EL SN CON DOS ADJETIVOS}

Los ejemplos que hemos ido manejando hasta el momento conciernen únicamente a un solo adjetivo. Sería oportuno examinar, a continuación, grupos más complejos, compuestos por dos adjetivos cuantitativos. En tales casos, los adjetivos se pueden configurar de formas muy distintas. Ejemplifiquémoslo:

su pintoresca figura majestuosa,

su majestuosa figura pintoresca, su majestuosa (y) pintoresca figura, su figura majestuosa (y) pintoresca,

los frondosos valles solitarios, los solitarios valles frondosos, los solitarios (y) frondosos valles, los valles frondosos (y) solitarios.

No obstante, existe una acusada tendencia, no sólo en español, de anteponer un tipo especial de adjetivos, llamados evaluativos, esto es, aquellos que se usan para expresar una evaluación o estimación, como bueno, feo, bonito, famoso, perfecto, impresionante, terrible, espantoso, majestuoso, etc. Valgan los sintagmas siguientes:

bonita pelota roja,

desmesurada crónica negra, impresionante revolución rápida, famosa isla solitaria, bellísima niña delgada.

Si las dos unidades aparecen conjuntamente a un lado del núcleo, y uno de ellos es un evaluativo, éste tiene que ir como primero en anteposición, por ejemplo:

la maravillosa larga jornada,

*la larga maravillosa jornada,

la extraordinaria dorada librería,

*la dorada extraordinaria librería, 
y como segundo en posición postnominal, como en:

la niña delgada bellísima,

*la niña bellísima delgada,

un coche rápido perfecto,

* un coche perfecto rápido.

Esta ordenación determinada se desprende del carácter semántico de los evaluativos. A saber, en vez de manifestar una cualidad concreta y objetiva del sustantivo, ofrecen una valoración o impresión que la persona o cosa significada producen en el hablante. Así, situándose en una posición extrema, inicial o final, los valorativos pueden dotar de su significado expresivo al conjunto conceptual integrado por el nombre y un adjetivo no evaluativo. Veámoslo otra vez en:

la niña delgada bellísima,

la bellísima delgada niña,

el doloroso largo silencio,

el silencio largo doloroso.

Este requisito lógico de colocar unidos al nombre términos que indican cualidades intrínsecas del referente, independientes de la voluntad del enunciante, es más general y se refiere, en la misma medida, también al polaco. Quizás por ello, sería mejor decir, p. ej., ten wspanialy dlugi spacer, ten czarujacy letni wieczór que ten dtugi wspaniaty spacer, ten letni czarujacy wieczór. Probablemente, la tarde en cuestión es encantadora por ser de verano y, también, el paseo parece magnífico por ser largo, y así el orden lógico va reflejado en el sintagmático.

Según se habrá notado, los complementos adjetivos que denotan rasgos físicos tan distintivos como color y dimensión tienden a ir en posición postnominal inmediata cuando hay más adjetivos implicados, p. ej.: libro amarillo sucio, *libro sucio amarillo; bonita pelota roja, pelota roja bonita, *pelota bonita roja. Es de notar que, por lo común, la secuencia de más de dos adjetivos postnominales es inusual en la lengua española. En este caso, los adjetivos evaluativos se suelen anteponer y el orden más normal de los otros, pospuestos, es en efecto aquel en el que un adjetivo de color va más cerca del nombre que el de dimensión; así: bonita pelota roja grande.

La configuración de los adjetivos se puede perturbar cuando intervienen al mismo tiempo dos adjetivos procedentes de distintas clases: uno cuantitativo y otro relacional. Aquí, el relacional siempre viene en posición postnominal, más cercana al nombre, y el cuantitativo se desplaza preferentemente al final o al principio de la expresión. Pongamos algunos ejemplos para ilustrarlo:

${ }^{5}$ Dada su semejanza categorial, los adjetivos de color y dimensión, al aparecer en una misma frase, pueden variar de posición, como en Dame el sombrero rojo redondo / Dame el sombrero redondo rojo. 


\author{
sólido fundamento intelectual, \\ fundamento intelecual sólido, \\ importante carácter simbólico, \\ carácter simbólico importante, \\ grave crisis polaca, \\ crisis polaca grave, \\ coherente programa electoral, \\ programa electoral coherente, \\ típico lugar turístico, \\ lugar turístico típico.
}

\title{
2.3. EL SN CON MÁS ADJETIVOS
}

$\mathrm{Al}$ menos como idealización, en posición prenominal las secuencias de adjetivos tienen como máximo un adjetivo cualitâtivo (dimensión, propiedad física, edad, etc.) y un número variable de miembros de otras clases. Sean los ejemplos:

mi posible futura amplia vivienda,

mi simpático alto amigo,

la supuesta única antigua amiga de mi madre.

Generalmente, los adjetivos modales pueden tanto preceder como seguir a los cualitativos, observación similar puede hacerse respecto del orden entre estos últimos y los evaluativos. Así:

el presunto delgado asesino / el delgado presunto asesino, la supuesta falsa declaración / la falsa supuesta declaración, mi verdadero único amigo / mi único verdadero amigo.

Desde luego, es perfectamente aceptable asimismo el desplazamiento de los términos no modales detrás del sustantivo. Por ello, obtenemos:

mi único amigo verdadero,

la supuesta declaración falsa, el presunto asesino delgado.

Conforme se apuntaba antes, cuando en la frase aparece un modificador relacional, va a tomar obligatoriamente su lugar acostumbrado postnominal. Veamos la posible alternancia dentro de la secuencia siguiente:

la supuesta vieja iglesia románica / la vieja supuesta iglesia románica. 


\section{OBSERVACIÓN FINAL}

Con este pequeño estudio nos hemos propuesto llamar la atención de los hablantes polacos de español como segunda lengua sobre el problema de la colocación de adjetivos calificativos dentro del SN español. Es, sin lugar a dudas, uno de los problemas coco de la gramática castellana que ahuyenta no sólo a los estudiantes polacos, sino también, y en primer lugar, a los usuarios de lenguas, como el ruso, alemán o inglés, que desconocen la libertad del orden de un elemento determinante y determinado a nivel sintagmático en sus propias lenguas. Huelga decir que la complejidad del sistema español, parecido en gran medida a los de otras lenguas románicas, es la herencia directa del latín que no tenía fijada la secuencia de los complementos adjetivos que calificaban al nombre. Además de esbozar algunas diferencias de uso resultantes del origen distinto del castellano y del polaco, hemos hecho unas observaciones referentes al comportamiento análogo de ciertos tipos de adjetivos en ambas lenguas. Así, hemos destacado que el problema en cuestión tiene una base semántica común y tiene que ser considerado mediante un análisis detallado de los contenidos escondidos bajo una forma adjetival determinada. La clasificación de los calificativos propuesta en páginas anteriores, y admitida por muchos lingüístas, constituye una aproximación a un examen más profundo de esta problemática bastante desatendida hasta ahora en la linguística hispánica.

\section{BIBLIOGRAFÍA}

Buttler D., Kurkowska H., Satkiewicz H. (1986), Kultura jezyka polskiego, Warszawa, PWN. Demonte V. (1998), Configuración e interpretación de los adjetivos del español: un enfoque minimista, en: Actas del $\mathrm{V}^{\circ}$ Coloquio Internacional de Linguíística Hispánica en la Universidad de Leipzig 1998, Peter Lang Verlag, (en prensa).

Moreno Cabrera, J.C. (1993), Curso universitario de lingüistica general l: Teoría de la gramática y sintaxis general, Madrid, Editorial Síntesis.

RAE (1973), Esbozo de una nueva gramática de la lengua española, Madrid, Espasa - Calpe.

Zierer E. (1974), The qualifying adjective in Spanish, The Hague - Paris, Mouton. 